\title{
Fuzzy Inventory Model for Deteriorating Items, with Time Depended Demand, Shortages, and Fully Backlogging
}

\author{
Wasim Akram Mandal \\ Beldanga D.H. Sr. Madrasah, Beldanga-742189, Murshidabad, WB, India \\ wasim0018@gmail.com \\ Sahidul Islam \\ Department of Mathematics, University of Kalyani, Kalyani, W.B, India \\ sahidul.math@gmail.com
}

\begin{abstract}
In this paper analyzes fuzzy inventory system for deterioration item with time depended demand. Shortages are allowed under fully backlogged. Fixed cost, deterioration cost, shortages cost, holding cost are the cost considered in this model. Fuzziness is applying by allowing the cost components (holding cost, deterioration, shortage cost, holding cost, etc). In fuzzy environment it considered all required parameter to be triangular fuzzy numbers. One numerical solution of the model is obtained to verify optimal solution. The purpose of the model is to minimize total cost function.
\end{abstract}

Keywords: Inventory, Deteriorating items, Fuzzy number, Shortages, Fully backlogged, Triangular fuzzy number.

\section{Introduction}

An inventory deal with decision that minimum the total average cost or maximize The total average profit. For this purpose the task is to construct a mathematical model of the real life Inventory system, such a mathematical model is based on various assumption and approximation

In a inventory model deterioration play an important role. Deterioration is defined as decay or damage in the quality of the inventory. Foods, Drugs, pharmaceuticals etc are deteriorating items. During inventory there have some losses of this deteriorating items, consequently this loss must be taken into account when analyzing the system. Shortages is also very important condition. There are several type of customer. At shortage period some customers are waiting for actual product and others do not it.

In ordinary inventory model it consider all parameter like shortage cost, holding cost, unit cost as fixed. But in real life situation it will have some little fluctuations. so consideration of fuzzy variables is more realistic.

The study of inventory model where demand rates varies with time is the last decades. Datta and pal investigated an inventory system with power demand pattern and deterioration. Park and Wang studied shortages and partial backlogging of items. Friedman (1978) presented continuous time inventory model with time varying demand. M. Roychowdhury and K.S Chaudhuri (1983) studied an order level inventory for deteriorating items with finite rate of replenishment. Ritchie (1984) studied in inventory model with linear increasing demand. Goswami, Chaudhuri (1991) discussed an 
inventory model with shortage. Gen et. Al. (1997) considered classical inventory model with Triangular fuzzy number. Yao and Lee (1998) considered an economic production quantity model in the fuzzy sense. Sujit Kumar De, P.K.Kundu and A.Goswami (2003) presented an economic production quantity inventory model involving fuzzy demand rate. J.K.Syde and L.A.Aziz (2007) applied sign distance method to fuzzy inventory model without shortage. D.Datta and Pravin Kumar published several paper of fuzzy inventory with or without shortage.

In this paper we first consider crisp inventory model with time depended demand where shortage are allowed and fully backlogged. Thereafter we developed fuzzy inventory model with fuzzy time depended demand rate under fully backlogged. All inventory cost parameters are fuzzyfied as triangular fuzzy number.

\section{Preliminaries}

For graded representation method to defuzzify, we need the following definitions, Definition2.1: A fuzzy set $\tilde{A}$ on the given universal set $\mathrm{X}$ is a set of order pairs, $\tilde{A}=\left\{\left(\mathrm{x}, \mu_{\mathrm{A}}(\mathrm{x})\right): \mathrm{x} \in \mathrm{X}\right\}$ where $\mu_{\mathrm{A}}(\mathrm{x}) \rightarrow[0,1]$ is called a membership function.

Definition2.2: The $\alpha$-cut of $\tilde{A}$, is defined by $\mathrm{A}_{\alpha}=\left\{\mathrm{x}: \mu_{\mathrm{A}}(\mathrm{x})=\alpha, \alpha \geq 0\right\}$

Definition2.3: $\tilde{A}$ is normal if there exists $\mathrm{x} \in \mathrm{X}$ such that $\mu_{\mathrm{A}}(\mathrm{x})=1$

Definition2.4: A triangular fuzzy number $\tilde{A}=(\mathrm{a}, \mathrm{b}, \mathrm{c})$ is represented with membership function $\tilde{A}$.

$\tilde{A}$ is defined as,

$$
\mu_{\mathrm{A}}(\mathrm{X})=\left\{\begin{array}{cc}
L(x)=\frac{x-a}{b-a}, & a \leq x \leq b \\
R(x)=\frac{c-x}{c-b}, & b \leq x \leq c \\
0, & \text { otherwise }
\end{array}\right.
$$

when $\mathrm{a}=\mathrm{b}=\mathrm{c}$, we have fuzzy point $(\mathrm{a}, \mathrm{a}, \mathrm{a})=\tilde{a}$. The family of all triangular fuzzy number on $\mathrm{R}$, denoted as $\mathrm{F}_{\mathrm{N}}=\{(\mathrm{a}, \mathrm{b}, \mathrm{c}), \mathrm{a}<\mathrm{b}<\mathrm{c}, \forall \mathrm{a}, \mathrm{b}, \mathrm{c} \in \mathrm{R}\}$. The $\alpha$-cut of $\tilde{A}=(\mathrm{a}, \mathrm{b}, \mathrm{c}) \in \mathrm{F}_{\mathrm{N}}, 0 \leq \alpha \leq 1$ is $\mathrm{A}(\alpha)=\left[\mathrm{A}_{\mathrm{L}}(\alpha), \mathrm{A}_{\mathrm{R}}(\alpha)\right]$ where $\mathrm{A}_{\mathrm{L}}(\alpha)=\mathrm{a}+(\mathrm{b}-\mathrm{a}) \alpha$ and $\mathrm{A}_{\mathrm{R}}(\alpha)=\mathrm{c}-(\mathrm{c}-\mathrm{b}) \alpha$ are the left and right end Point of $A(\alpha)$.

Definition2.5: If $\tilde{A}=(\mathrm{a}, \mathrm{b}, \mathrm{c})$ is a triangular fuzzy number then the graded mean integration of $\tilde{A}$ is defined as,

$$
\begin{aligned}
\mathrm{P}(\widetilde{A}) & =\frac{\int_{0}^{W_{A}} h\left(\frac{L^{-1}(\mathrm{~h})+R^{-1}(\mathrm{~h})}{2}\right) d h}{\int_{0}^{W_{A}} h d h}, \quad\left(0 \leq \mathrm{h} \leq \mathrm{W}_{\mathrm{A}} \text { and } 0 \leq \mathrm{W}_{\mathrm{A}} \leq 1\right) \\
\mathrm{P} \widetilde{(A)} & =\frac{\int_{0}^{1} h\left[\frac{a+(b-a) h+c-(c-b) h] d h}{2}\right]}{2 \int_{0}^{1} h d h} \\
& =\frac{a+4 b+c}{6}
\end{aligned}
$$


Suppose $\tilde{a}=\left(\mathrm{a}_{1}, \mathrm{a}_{2}, \mathrm{a}_{3}\right)$ and $\tilde{b}=\left(\mathrm{b}_{1}, \mathrm{~b}_{2}, \mathrm{~b}_{3}\right)$ are two fuzzy triangular number then

(1) $\tilde{a}+\tilde{b}=\left(\mathrm{a}_{1}, \mathrm{a}_{2}, \mathrm{a}_{3}\right)+\left(\mathrm{b}_{1}, \mathrm{~b}_{2}, \mathrm{~b}_{3}\right)=\left(\mathrm{a}_{1}+\mathrm{b}_{1}, \mathrm{a}_{2}+\mathrm{b}_{2}, \mathrm{a}_{3}+\mathrm{b}_{3}\right)$.

(2) $\quad \tilde{a}-\tilde{b}=\left(\mathrm{a}_{1}, \mathrm{a}_{2}, \mathrm{a}_{3}\right)-\left(\mathrm{b}_{1}, \mathrm{~b}_{2}, \mathrm{~b}_{3}\right)=\left(\mathrm{a}_{1}-\mathrm{b}_{1}, \mathrm{a}_{2}-\mathrm{b}_{2}, \mathrm{a}_{3}-\mathrm{b}_{3}\right)$.

(3) $\quad \tilde{a} \times \tilde{b}=\left(\mathrm{a}_{1}, \mathrm{a}_{2}, \mathrm{a}_{3}\right) \times\left(\mathrm{b}_{1}, \mathrm{~b}_{2}, \mathrm{~b}_{3}\right)=\left(\mathrm{a}_{1} \mathrm{~b}_{1}, \mathrm{a}_{2} \mathrm{~b}_{2}, \mathrm{a}_{3} \mathrm{~b}_{3}\right)$.

(4) $\frac{1}{\tilde{b}}=\left(\frac{1}{b_{3}}, \frac{1}{b_{2}}, \frac{1}{b_{1}}\right)$ where $b_{1}, b_{1}, b_{1}$ are all non zero positive real number, then

$$
\frac{\tilde{a}}{\tilde{b}}=\left(\frac{\mathrm{a}_{1}}{b_{3}}, \frac{\mathrm{a}_{2}}{b_{2}}, \frac{\mathrm{a}_{3}}{b_{1}}\right) \text {. }
$$

(5) Let $\mathrm{k} \in \mathrm{R}$, then $\mathrm{k} \tilde{a}=\mathrm{k}\left(\mathrm{a}_{1}, \mathrm{a}_{2}, \mathrm{a}_{3}\right)=\left(\mathrm{ka}_{1}, \mathrm{ka}_{2}, \mathrm{ka} \mathrm{a}_{3}\right)$ for $\mathrm{k} \geq 0$.

\subsection{Notation}

$\mathbf{I}(\mathbf{t})=$ Inventory level at any time, $\mathrm{t} \geq 0$.

$\mathrm{T}:$ Cycle of length.

$\mathrm{t}_{\mathrm{w}}$ : Time point, when demand rate start with (c-dt).

$\mathrm{t}_{1}$ : Time point when stock level reaches to zero.

$\mathrm{c}_{1}$ :Fixed cost.

$\mathrm{c}_{2}$ :Shortages cost per unit.

$\mathrm{c}_{3}$ :Deteriorating cost per unit.

$\mathrm{c}_{4}$ :Holding cost per unit.

Q:Highest stock level at the beginning of the cycle.

$\operatorname{TAC}\left(\mathrm{t}_{\mathrm{w}}, \mathrm{t}_{1}\right)$ :Total average cost per unit.

$\tilde{c}_{1}=$ Fuzzy fixed cost.

$\tilde{c}_{2}=$ Fuzzy shortage cost per unit.

$\tilde{c}_{3}=$ Fuzzy deteriorating cost per unit.

$\tilde{c}_{4}=$ Fuzzy holding cost per unit.

$\left.T A \widetilde{C\left(\mathrm{t}_{\mathrm{w}}\right.}, \mathrm{t}_{1}\right)=$ Fuzzy total cost per unit.

\subsection{Assumption}

a: The inventory system involves only one item.

b: The replenishment occur instantaneously at infinite rate.

c: $\quad$ The lead time is negligible.

$\mathrm{d}$ : $\quad$ Demand rate is time depended, we assume it $(\mathrm{a}+\mathrm{bt})$ in $0 \leq \mathrm{t} \leq \mathrm{t}_{\mathrm{w}}$ and (c-dt) in $\mathrm{t}_{\mathrm{w}} \leq \mathrm{t} \leq \mathrm{t}_{1}$.

e: $\quad 2 \theta$ is deterioration rate per unit time per cycle, $\theta$ is constant.

g: At time $\mathrm{t}=\mathrm{t}_{\mathrm{w}}$ it should be $\left(\mathrm{a}+\mathrm{bt}_{\mathrm{w}}\right)=\left(\mathrm{c}-\mathrm{dt}_{\mathrm{w}}\right)$. 


\subsection{Model Development (Crisp Model)}

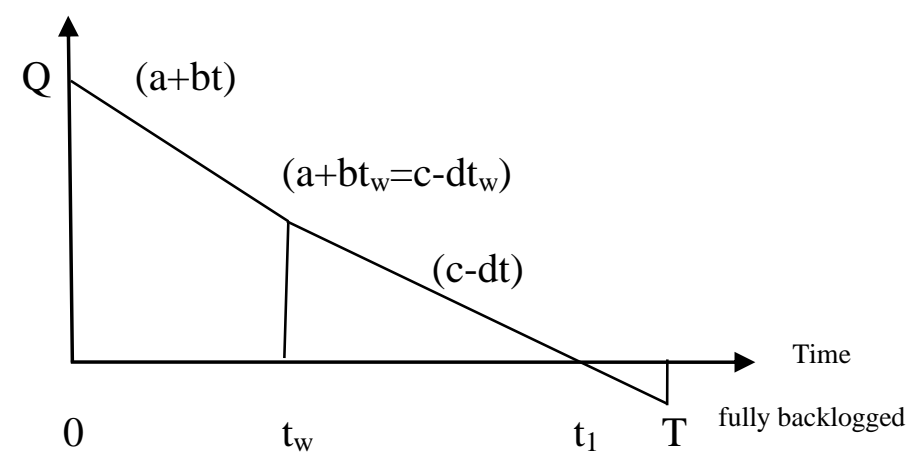

During $0 \leq t \leq t_{\mathrm{w}}$ the inventory level decrease due to customer demand (rate of demand $=a+b t$ ) and deteriorating items. During $t_{w} \leq t \leq t_{1}$ the inventory level decrease due to customer demand (rate of demand $=\mathrm{c}-\mathrm{dt}$ ), deteriorating items and reaches to zero at $\mathrm{t}=\mathrm{t}_{1}$. In the time interval $\mathrm{t}_{1} \leq \mathrm{t} \leq \mathrm{T}$ shortages with fully backlogged allowed.

The differential equation describing $\mathrm{I}(\mathrm{t})$ as follows

$$
\frac{d I(t)}{d t}+2 \theta I(t)=-(\mathrm{a}+\mathrm{bt}), \quad 0 \leq \mathrm{t} \leq \mathrm{t}_{\mathrm{w}}
$$

With boundary condition $\mathrm{I}(0)=\mathrm{Q}$.

$$
\frac{d I(t)}{d t}+2 \theta I(t)=-(\mathrm{c}-\mathrm{dt}), \quad \mathrm{t}_{\mathrm{w}} \leq \mathrm{t} \leq \mathrm{t}_{1}
$$

With boundary condition $\mathrm{I}\left(\mathrm{t}_{1}\right)=0$

$$
\frac{d I(t)}{d t}=-(c-d t), \quad \mathrm{t}_{1} \leq \mathrm{t} \leq \mathrm{T}
$$

With boundary condition $\mathrm{I}\left(\mathrm{t}_{1}\right)=0$.

From (3.1) we get,

$$
\mathrm{I}(\mathrm{t})=\mathrm{Q}\left(1-\theta t^{2}\right)-\left(1-\theta t^{2}\right)\left(\mathrm{at}+\frac{b t^{2}}{2}\right)
$$

From (3.2) we get,

$$
\mathrm{I}(\mathrm{t})=\mathrm{c}\left(1-\theta t^{2}\right)\left(t_{1}-\mathrm{t}\right)-\frac{d}{2}\left(t_{1}^{2}-t^{2}\right)\left(1-\theta t^{2}\right)
$$

From (3.3) we get,

$$
\mathrm{I}(\mathrm{t})=\mathrm{c}\left(t_{1}-\mathrm{t}\right)-\frac{d}{2}\left(t_{1}^{2}-t^{2}\right)
$$

So the fixed cost per cycle is,

$$
\mathrm{FC}=\mathrm{c}_{1}
$$

Shortage cost per cycle is,

$$
\begin{aligned}
\mathrm{Sc} & =-\mathrm{c}_{2} \int_{t_{1}}^{T} I(t) \mathrm{dt} \\
& =-\mathrm{c}_{2}\left[\mathrm{c}\left(\mathrm{t}_{1} \mathrm{~T}-\frac{T^{2}}{2}\right)-\frac{C t_{1}{ }^{2}}{2}-\frac{d}{2}\left(t_{1}{ }^{2} \mathrm{~T}-\frac{T^{3}}{3}\right)+\frac{d t_{1}{ }^{3}}{3}\right] .
\end{aligned}
$$


Deteriorating cost per cycle is,

$$
\begin{aligned}
& \mathrm{DC}=\mathrm{c}_{3} \int_{0}^{t_{w}}(a+b t) I(t) d t+\mathrm{c}_{3} \int_{t_{w}}^{t_{1}}(c-d t) I(t) d t \\
& =\mathrm{c}_{3}\left[{\mathrm{Q} \alpha \mathrm{t}_{\mathrm{w}^{-}}} \frac{Q a \theta t_{w}{ }^{3}}{3}-\frac{a^{2} t_{w}{ }^{2}}{2}-\frac{a b t_{w}{ }^{3}}{6}+\frac{a^{2} \theta t_{w}{ }^{4}}{4}+\frac{a b \theta t_{w}{ }^{5}}{10}+\frac{b Q t_{w}{ }^{2}}{2}-\frac{Q b \theta t_{w}{ }^{4}}{4}-\frac{a b t_{w}{ }^{3}}{3}-\right. \\
& \frac{b^{2} t_{w}{ }^{4}}{8}+\frac{a b \theta t_{w}{ }^{5}}{5}+\frac{b^{2} \theta t_{w}{ }^{6}}{12}+c^{2}\left\{\mathrm{t}_{1}\left(\mathrm{t}_{1}-\mathrm{t}_{\mathrm{w}}\right)-\frac{t_{1}{ }^{2}-t_{w}{ }^{2}}{2}-\frac{\theta t_{1}\left(t_{1}{ }^{3}-t_{w}{ }^{3}\right)}{3}+\frac{\theta\left(t_{1}{ }^{4}-t_{w}{ }^{4}\right)}{4}\right\}- \\
& \operatorname{cd}\left\{\frac{t_{1}\left(t_{1}{ }^{2}-t_{w}{ }^{2}\right)}{2}-\frac{\left(t_{1}{ }^{3}-t_{w}{ }^{3}\right)}{3}-\frac{\theta t_{1}\left(t_{1}{ }^{4}-t_{w}{ }^{4}\right)}{4}+\frac{\theta\left(t_{1}{ }^{5}-t_{w}{ }^{5}\right)}{5}\right\}-\frac{c d}{2}\left\{t_{1}{ }^{2}\left(\mathrm{t}_{1}-\mathrm{t}_{\mathrm{w}}\right)-\right. \\
& \left.\frac{\left(t_{1}{ }^{3}-t_{w}{ }^{3}\right)}{3}-\frac{\theta t_{1}{ }^{2}\left(t_{1}{ }^{3}-t_{w}{ }^{3}\right)}{3}-\frac{\theta\left(t_{1}{ }^{5}-t_{w}{ }^{5}\right)}{5}\right\}+\frac{d^{2}}{2}\left\{\frac{t_{1}{ }^{2}\left(t_{1}{ }^{2}-t_{w}{ }^{2}\right)}{2}-\frac{\left(t_{1}{ }^{4}-t_{w}{ }^{4}\right)}{4}-\right. \\
& \left.\left.\frac{\theta t_{1}{ }^{2}\left(t_{1}{ }^{4}-t_{w}{ }^{4}\right)}{4}+\frac{\theta\left(t_{1}{ }^{6}-t_{w}{ }^{6}\right)}{6}\right\}\right] \text {. }
\end{aligned}
$$

Holding cost per cycle is,

$$
\begin{aligned}
& \mathrm{HC}=\mathrm{c}_{4} \int_{0}^{t_{w}} I(t) d t+\mathrm{c}_{4} \int_{t_{w}}^{t_{1}} I(t) d t \\
& =\mathrm{c}_{4}\left[\mathrm{Q}\left(\mathrm{t}_{\mathrm{w}}-\frac{Q t_{w}{ }^{3}}{3}\right)-\left(\frac{a t_{w}{ }^{2}}{2}+\frac{b t_{w}{ }^{3}}{6}\right)+\theta\left(\frac{a t_{w}{ }^{4}}{4}+\frac{b t_{w}{ }^{5}}{10}\right)+\mathrm{c}\left\{\mathrm{t}_{1}\left(\mathrm{t}_{1}-\mathrm{t}_{\mathrm{w}}\right)-\frac{t_{1}{ }^{2}-t_{w}{ }^{2}}{2}\right\}-\right. \\
& \mathrm{c} \theta\left\{\frac{t_{1}{ }^{3}\left(t_{1}{ }^{3}-t_{w}{ }^{3}\right)}{3}-\frac{\left(t_{1}{ }^{4}-t_{w}{ }^{4}\right)}{4}\right\} \quad-\frac{d}{2}\left\{t_{1}{ }^{2}\left(t_{1}-t_{w}\right)-\frac{t_{1}{ }^{3}-t_{w}{ }^{3}}{3}\right\} \\
& \left.+\frac{d \theta}{2}\left\{\frac{\left.t_{1}^{2(} t_{1}^{3}-t_{w}{ }^{3}\right)}{3}-\frac{\left.t_{1}^{5}-t_{w}^{5}\right)}{5}\right\}\right] \text {. }
\end{aligned}
$$

So total average cost per cycle is,

$$
\begin{aligned}
& \mathrm{TAC}\left(\mathrm{t}_{1}, \mathrm{t}_{\mathrm{w}}\right)=\frac{1}{T}[\mathrm{FC}+\mathrm{SC}+\mathrm{DC}+\mathrm{HC}] \\
& =\frac{1}{T}\left[\left[\mathrm{c}_{1}-\mathrm{c}_{2}\left\{\mathrm{c}\left(\mathrm{t}_{1} \mathrm{~T}-\frac{T^{2}}{2}\right)-\frac{C t_{1}{ }^{2}}{2}-\frac{d}{2}\left(t_{1}{ }^{2} \mathrm{~T}-\frac{T^{3}}{3}\right)+\frac{d t_{1}{ }^{3}}{3}\right\}+\mathrm{c}_{3}\left[\mathrm{Q} \alpha \mathrm{t}_{\mathrm{w}^{-}} \frac{Q a \theta t_{w^{3}}}{3}-\frac{a^{2} t_{w^{2}}}{2}-\right.\right.\right. \\
& \frac{a b t_{w}{ }^{3}}{6}+\frac{a^{2} \theta t_{w}{ }^{4}}{4}+\frac{a b \theta t_{w}{ }^{5}}{10}+\frac{b Q t_{w}{ }^{2}}{2}-\frac{Q b \theta t_{w}{ }^{4}}{4}-\frac{a b t_{w}{ }^{3}}{3}-\frac{b^{2} t_{w}{ }^{4}}{8}+\frac{a b \theta t_{w}{ }^{5}}{5}+\frac{b^{2} \theta t_{w}{ }^{6}}{12} \\
& +\mathrm{c}^{2}\left\{\mathrm{t}_{1}\left(\mathrm{t}_{1}-\mathrm{t}_{\mathrm{w}}\right)-\frac{t_{1}{ }^{2}-t_{w}{ }^{2}}{2}-\frac{\theta t_{1}\left(t_{1}{ }^{3}-t_{w}{ }^{3}\right)}{3}+\frac{\theta\left(t_{1}{ }^{4}-t_{w}{ }^{4}\right)}{4}\right\}-\operatorname{cd}\left\{\frac{t_{1}\left(t_{1}{ }^{2}-t_{w}{ }^{2}\right)}{2}-\frac{\left(t_{1}{ }^{3}-t_{w}{ }^{3}\right)}{3}-\right. \\
& \left.\frac{\theta t_{1}\left(t_{1}{ }^{4}-t_{w}{ }^{4}\right)}{4}+\frac{\theta\left(t_{1}{ }^{5}-t_{w}{ }^{5}\right)}{5}\right\}-\frac{c d}{2}\left\{t_{1}{ }^{2}\left(\mathrm{t}_{1}-\mathrm{t}_{\mathrm{w}}\right)-\frac{\left(t_{1}{ }^{3}-t_{w^{3}}\right)}{3}-\frac{\theta t_{1}{ }^{2}\left(t_{1}{ }^{3}-t_{w}{ }^{3}\right)}{3}-\right. \\
& \left.\left.\frac{\theta\left(t_{1}{ }^{5}-t_{w}{ }^{5}\right)}{5}\right\}+\frac{d^{2}}{2}\left\{\frac{t_{1}{ }^{2}\left(t_{1}{ }^{2}-t_{w}{ }^{2}\right)}{2}-\frac{\left(t_{1}{ }^{4}-t_{w}{ }^{4}\right)}{4}-\frac{\theta t_{1}{ }^{2}\left(t_{1}{ }^{4}-t_{w}{ }^{4}\right)}{4}+\frac{\theta\left(t_{1}{ }^{6}-t_{w}{ }^{6}\right)}{6}\right\}\right]+\mathrm{c}_{4}\left[\mathrm { Q } \left(\mathrm{t}_{\mathrm{w}}\right.\right. \\
& \left.-\frac{Q t_{w}{ }^{3}}{3}\right)-\left(\frac{a t_{w}{ }^{2}}{2}+\frac{b t_{w}{ }^{3}}{6}\right)+\theta\left(\frac{a t_{w}{ }^{4}}{4}+\frac{b t_{w}{ }^{5}}{10}\right)+\mathrm{c}\left\{\mathrm{t}_{1}\left(\mathrm{t}_{1}-\mathrm{t}_{\mathrm{w}}\right)-\frac{t_{1}{ }^{2}-t_{w}{ }^{2}}{2}\right\}-\mathrm{c} \theta\left\{\frac{t_{1}{ }^{3}\left(t_{1}{ }^{3}-t_{w}{ }^{3}\right)}{3}\right. \\
& \left.\left.\left.-\frac{\left(t_{1}{ }^{4}-t_{w}{ }^{4}\right)}{4}\right\}-\frac{d}{2}\left\{t_{1}{ }^{2}\left(t_{1}-t_{w}\right)-\frac{t_{1}{ }^{3}-t_{w}{ }^{3}}{3}\right\}+\frac{d \theta}{2}\left\{\frac{\left.t_{1}{ }^{2(} t_{1}{ }^{3}-t_{w}{ }^{3}\right)}{3}-\frac{\left.t_{1}{ }^{5}-t_{w}{ }^{5}\right)}{5}\right\}\right]\right] .
\end{aligned}
$$

For minimum cost it should be,

$$
\frac{\partial T A C\left(t_{w}, t_{1}\right)}{\partial t_{w}}=0, \frac{\partial T A C\left(t_{w}, t_{1}\right)}{\partial t_{1}}=0
$$

Provided it satisfies,

$$
\begin{gathered}
\frac{\partial^{2} \mathrm{TAC}\left(\mathrm{t}_{\mathrm{w}}, \mathrm{t}_{1}\right)}{\partial t_{w}{ }^{2}}>0, \quad \frac{\partial^{2} \mathrm{TAC}\left(\mathrm{t}_{\mathrm{w}}, \mathrm{t}_{1}\right)}{\partial t_{1}{ }^{2}}>0 \\
\text { and } \quad\left[\frac{\partial^{2} \mathrm{TAC}\left(\mathrm{t}_{w}, \mathrm{t}_{1}\right)}{\partial t_{w}{ }^{2}}\right]\left[\frac{\partial^{2} \mathrm{TAC}\left(\mathrm{t}_{\mathrm{w}}, \mathrm{t}_{1}\right)}{\partial t_{w}{ }^{2}}\right]-\left[\frac{\partial^{2} \mathrm{TAC}\left(\mathrm{t}_{\mathrm{w}}, \mathrm{t}_{1}\right)}{\partial t_{w} \partial t_{1}}\right]>0 .
\end{gathered}
$$




\subsection{Fuzzy Model}

Due to uncertainly lets us assume that,

$\widetilde{c_{1}}=\left(\mathrm{c}_{1}{ }^{1}, \mathrm{c}_{1}{ }^{2}, \mathrm{c}_{1}{ }^{3}\right), \quad \widetilde{c_{2}}=\left(\mathrm{c}_{2}{ }^{1}, \mathrm{c}_{2}{ }^{2}, \mathrm{c}_{2}{ }^{3}\right), \widetilde{c_{3}}=\left(\mathrm{c}_{3}{ }^{1}, \mathrm{c}_{3}{ }^{2}, \mathrm{c}_{3}{ }^{3}\right), \widetilde{c_{4}}=\left(\mathrm{c}_{4}{ }^{1}, \mathrm{c}_{4}{ }^{2}, \mathrm{c}_{4}{ }^{3}\right)$ be triangular fuzzy number then the total average cost is given by,

$\left.T \widetilde{A C\left(t_{w}\right.}, t_{1}\right)=\frac{1}{T}\left[\left[\widetilde{c_{1}}-\widetilde{c_{2}}\left\{\mathrm{c}\left(\mathrm{t}_{1} \mathrm{~T}-\frac{T^{2}}{2}\right)-\frac{C t_{1}{ }^{2}}{2}-\frac{d}{2}\left(t_{1}{ }^{2} \mathrm{~T}-\frac{T^{3}}{3}\right)+\frac{d t_{1}{ }^{3}}{3}\right\}+\widetilde{c_{3}}\left[\mathrm{Q} \alpha \mathrm{t}_{\mathrm{w}^{-}} \frac{Q a \theta t_{w}{ }^{3}}{3}-\frac{a^{2} t_{w}{ }^{2}}{2}-\right.\right.\right.$ $\frac{a b t_{w}{ }^{3}}{6}+\frac{a^{2} \theta t_{w}{ }^{4}}{4}+\frac{a b \theta t_{w}{ }^{5}}{10}+\frac{b Q t_{w}{ }^{2}}{2}-\frac{Q b \theta t_{w}{ }^{4}}{4}-\frac{a b t_{w}{ }^{3}}{3}-\frac{b^{2} t_{w}{ }^{4}}{8}+\frac{a b \theta t_{w}{ }^{5}}{5}+\frac{b^{2} \theta t_{w}{ }^{6}}{12}+c^{2}\left\{\mathrm{t}_{1}\left(\mathrm{t}_{1}-\mathrm{t}_{\mathrm{w}}\right)-\frac{t_{1}{ }^{2}-t_{w}{ }^{2}}{2}-\right.$ $\left.\frac{\theta t_{1}\left(t_{1}{ }^{3}-t_{w}{ }^{3}\right)}{3}+\frac{\theta\left(t_{1}{ }^{4}-t_{w}{ }^{4}\right)}{4}\right\}-\operatorname{cd}\left\{\frac{t_{1}\left(t_{1}{ }^{2}-t_{w}{ }^{2}\right)}{2}-\frac{\left(t_{1}{ }^{3}-t_{w}{ }^{3}\right)}{3}-\frac{\theta t_{1}\left(t_{1}{ }^{4}-t_{w}{ }^{4}\right)}{4}+\frac{\theta\left(t_{1}{ }^{5}-t_{w}{ }^{5}\right)}{5}\right\}-\frac{c d}{2}\left\{t_{1}{ }^{2}\left(\mathrm{t}_{1}-\right.\right.$ $\left.\left.\mathrm{t}_{\mathrm{w}}\right)-\frac{\left(t_{1}{ }^{3}-t_{w}{ }^{3}\right)}{3}-\frac{\theta t_{1}{ }^{2}\left(t_{1}{ }^{3}-t_{w}{ }^{3}\right)}{3}-\frac{\theta\left(t_{1}{ }^{5}-t_{w}{ }^{5}\right)}{5}\right\}+\frac{d^{2}}{2}\left\{\frac{t_{1}{ }^{2}\left(t_{1}{ }^{2}-t_{w}{ }^{2}\right)}{2}-\frac{\left(t_{1}{ }^{4}-t_{w}{ }^{4}\right)}{4}-\right.$ $\left.\left.\frac{\theta t_{1}{ }^{2}\left(t_{1}{ }^{4}-t_{w}{ }^{4}\right)}{4}+\frac{\theta\left(t_{1}{ }^{6}-t_{w}{ }^{6}\right)}{6}\right\}\right]+\widetilde{c_{4}}\left[\mathrm{Q}\left(\mathrm{t}_{\mathrm{w}}-\frac{Q t_{w}{ }^{3}}{3}\right)-\left(\frac{a t_{w}{ }^{2}}{2}+\frac{b t_{w}{ }^{3}}{6}\right)+\theta\left(\frac{a t_{w}{ }^{4}}{4}+\frac{b t_{w}{ }^{5}}{10}\right)+\mathrm{c}\left\{\mathrm{t}_{1}\left(\mathrm{t}_{1}-\mathrm{t}_{\mathrm{w}}\right)-\right.\right.$ $\left.\frac{t_{1}{ }^{2}-t_{w}{ }^{2}}{2}\right\}-c \theta\left\{\frac{t_{1}{ }^{3}\left(t_{1}{ }^{3}-t_{w}{ }^{3}\right)}{3}-\frac{\left(t_{1}{ }^{4}-t_{w}{ }^{4}\right)}{4}\right\}-\frac{d}{2}\left\{t_{1}{ }^{2}\left(t_{1}-t_{w}\right)-\frac{t_{1}{ }^{3}-t_{w}{ }^{3}}{3}\right\}+\frac{d \theta}{2}\left\{\frac{t_{1}{ }^{2}\left(t_{1}{ }^{3}-t_{w}{ }^{3}\right)}{3}-\right.$ $\left.\left.\left.\frac{\left.t_{1}^{5}-t_{w}^{5}\right)}{5}\right\}\right]\right]$.

We defuzzifi the fuzzy total cost $\left.T \widetilde{A C\left(t_{w}\right.}, t_{1}\right)$ by graded mean representation method as follows,

$$
\left.T \widetilde{A C(} t_{w}, t_{1}\right)=\frac{1}{6}\left[\widetilde{T A C^{1}}\left(\mathrm{t}_{\mathrm{w}}, \mathrm{t}_{1}\right), \widetilde{T A C^{2}}\left(\mathrm{t}_{\mathrm{w}}, \mathrm{t}_{1}\right), \widetilde{T A C^{3}}\left(\mathrm{t}_{\mathrm{w}}, \mathrm{t}_{1}\right)\right]
$$

Where

$\widetilde{T_{A C}}\left(\mathrm{t}_{\mathrm{w}}, \mathrm{t}_{1}\right)=\frac{1}{T}\left[\left[\widetilde{c_{1}{ }^{r}}-\widetilde{c_{2}{ }^{r}}\left\{\mathrm{c}\left(\mathrm{t}_{1} \mathrm{~T}-\frac{T^{2}}{2}\right)-\frac{C t_{1}{ }^{2}}{2}-\frac{d}{2}\left(t_{1}{ }^{2} \mathrm{~T}-\frac{T^{3}}{3}\right)+\frac{d t_{1}{ }^{3}}{3}\right\}+\widetilde{{c_{3}}^{r}}\left[\mathrm{Q} \alpha \mathrm{t}_{\mathrm{w}^{-}} \frac{Q a \theta t_{w}{ }^{3}}{3}-\frac{a^{2} t_{w}{ }^{2}}{2}\right.\right.\right.$ $-\frac{a b t_{w}{ }^{3}}{6}+\frac{a^{2} \theta t_{w}{ }^{4}}{4}+\frac{a b \theta t_{w}{ }^{5}}{10}+\frac{b Q t_{w}{ }^{2}}{2}-\frac{Q b \theta t_{w}{ }^{4}}{4}-\frac{a b t_{w}{ }^{3}}{3}-\frac{b^{2} t_{w}{ }^{4}}{8}+\frac{a b \theta t_{w}{ }^{5}}{5}+\frac{b^{2} \theta t_{w}{ }^{6}}{12}+c^{2}\left\{\mathrm{t}_{1}\left(\mathrm{t}_{1}-\mathrm{t}_{\mathrm{w}}\right)-\frac{t_{1}{ }^{2}-t_{w}{ }^{2}}{2}\right.$ $\left.-\frac{\theta t_{1}\left(t_{1}{ }^{3}-t_{w}{ }^{3}\right)}{3}+\frac{\theta\left(t_{1}{ }^{4}-t_{w}{ }^{4}\right)}{4}\right\}-\operatorname{cd}\left\{\frac{t_{1}\left(t_{1}{ }^{2}-t_{w}{ }^{2}\right)}{2}-\frac{\left(t_{1}{ }^{3}-t_{w}{ }^{3}\right)}{3}-\frac{\theta t_{1}\left(t_{1}{ }^{4}-t_{w}{ }^{4}\right)}{4}+\frac{\theta\left(t_{1}{ }^{5}-t_{w}{ }^{5}\right)}{5}\right\}-\frac{c d}{2}\left\{t_{1}{ }^{2}\left(\mathrm{t}_{1}-\right.\right.$ $\left.\left.\mathbf{t}_{\mathrm{w}}\right)-\frac{\left(t_{1}{ }^{3}-t_{w}{ }^{3}\right)}{3}-\frac{\theta t_{1}{ }^{2}\left(t_{1}{ }^{3}-t_{w}{ }^{3}\right)}{3}-\frac{\theta\left(t_{1}{ }^{5}-t_{w}{ }^{5}\right)}{5}\right\}+\frac{d^{2}}{2}\left\{\frac{t_{1}{ }^{2}\left(t_{1}{ }^{2}-t_{w}{ }^{2}\right)}{2}-\frac{\left(t_{1}{ }^{4}-t_{w}{ }^{4}\right)}{4}-\right.$ $\left.\left.\frac{\theta t_{1}{ }^{2}\left(t_{1}{ }^{4}-t_{w}{ }^{4}\right)}{4}+\frac{\theta\left(t_{1}{ }^{6}-t_{w}{ }^{6}\right)}{6}\right\}\right]+\widetilde{c_{4}}\left[\mathrm{Q}\left(\mathrm{t}_{\mathrm{w}}-\frac{Q t_{w}{ }^{3}}{3}\right)-\left(\frac{a t_{w}{ }^{2}}{2}+\frac{b t_{w}{ }^{3}}{6}\right)+\theta\left(\frac{a t_{w}{ }^{4}}{4}+\frac{b t_{w}{ }^{5}}{10}\right)+\mathrm{c}\left\{\mathrm{t}_{1}\left(\mathrm{t}_{1}-\mathrm{t}_{\mathrm{w}}\right)-\right.\right.$ $\left.\frac{t_{1}{ }^{2}-t_{w}{ }^{2}}{2}\right\}-c \theta\left\{\frac{t_{1}{ }^{3}\left(t_{1}{ }^{3}-t_{w}{ }^{3}\right)}{3}-\frac{\left(t_{1}{ }^{4}-t_{w}{ }^{4}\right)}{4}\right\}-\frac{d}{2}\left\{t_{1}{ }^{2}\left(t_{1}-t_{w}\right)-\frac{t_{1}{ }^{3}-t_{w}{ }^{3}}{3}\right\}+\frac{d \theta}{2}\left\{\frac{t_{1}{ }^{2}\left(t_{1}{ }^{3}-t_{w}{ }^{3}\right)}{3}-\right.$ $\left.\left.\left.\frac{\left.t_{1}{ }^{5}-t_{w}{ }^{5}\right)}{5}\right\}\right]\right] . \quad \mathrm{r}=1,2,3$.

$\left.T \widetilde{A C(} t_{1}\right)=\frac{1}{6}\left[\widetilde{T A C}_{1}\left(\mathrm{t}_{\mathrm{w}}, \mathrm{t}_{1}\right)+4 \widetilde{T A C_{2}}\left(\mathrm{t}_{\mathrm{w}}, \mathrm{t}_{1}\right)+\widetilde{T A C_{3}}\left(\mathrm{t}_{\mathrm{w}}, \mathrm{t}_{1}\right)\right]$ 
For minimum cost it should be,

$$
\frac{\partial T A \widetilde{C\left(\mathrm{t}_{\mathrm{w}}, \mathrm{t}_{1}\right)}}{\partial t_{w}}=0, \frac{\partial T A \widetilde{C\left(\mathrm{t}_{\mathrm{w}}, \mathrm{t}_{1}\right)}}{\partial t_{1}}=0
$$

Provided it satisfies,

$$
\begin{gathered}
\frac{\left.\partial^{2} T A \widetilde{C\left(\mathrm{t}_{\mathrm{w}}\right.}, \mathrm{t}_{1}\right)}{\partial t_{w}{ }^{2}}>0, \quad \frac{\partial^{2} T A \widetilde{C\left(\mathrm{t}_{\mathrm{w}}, \mathrm{t}_{1}\right)}}{\partial t_{1}{ }^{2}}>0 \\
\text { and } \quad\left[\frac{\left.\partial^{2} T A \widetilde{C\left(\mathrm{t}_{\mathrm{w}}\right.}, \mathrm{t}_{1}\right)}{\partial t_{w}{ }^{2}}\right]\left[\frac{\partial^{2} T A \widetilde{C\left(\mathrm{t}_{\mathrm{w}}, \mathrm{t}_{1}\right)}}{\partial t_{1}{ }^{2}}\right]-\left[\frac{\partial^{2} T A \widetilde{C\left(\mathrm{t}_{\mathrm{w}}, \mathrm{t}_{1}\right)}}{\partial t_{w} \partial t_{1}}\right]>0 .
\end{gathered}
$$

\section{Numerical Solution}

For crisp model: Let us take the in-put value:

\begin{tabular}{|c|c|c|c|c|c|c|c|c|c|c|}
\hline $\mathrm{C}_{1}$ & $\mathrm{C}_{2}$ & $\mathrm{C}_{3}$ & $\mathrm{C}_{4}$ & $\theta$ & $\mathrm{Q}$ & $\mathrm{a}$ & $\mathrm{b}$ & $\mathrm{c}$ & $\mathrm{d}$ & $\mathrm{T}$ \\
\hline 500 & 1 & 2 & 1 & 0.1 & 100 & 5 & 10 & 7 & 10 & 2 \\
\hline
\end{tabular}

And the out-put value:

For fuzzy model:

\begin{tabular}{|c|c|c|}
\hline $\mathrm{t}_{\mathrm{w}}$ & $\mathrm{t}_{1}$ & $\mathrm{TAC}\left(\mathrm{t}_{\mathrm{w}}, \mathrm{t}_{1}\right)$ \\
\hline 0.100 & 0.266 & 266.162 \\
\hline
\end{tabular}

$$
\begin{aligned}
& \widetilde{c_{1}}=(450,500,550), \widetilde{c_{2}}=(0.9,1 \cdot 0,1 \cdot 1), \widetilde{c_{3}}=(1 \cdot 8,2 \cdot 0,2 \cdot 2), \\
& \widetilde{c_{4}}=(0.9,1 \cdot 0,1 \cdot 1),
\end{aligned}
$$

The solution of fuzzy model by graded mean representation is,

(1) When $\widetilde{c_{1}}, \widetilde{c_{2}}, \widetilde{c_{3}}, \widetilde{c_{4}}$ are all triangular fuzzy numbers then,

$\operatorname{TAC}\left(\mathrm{t}_{\mathrm{w}}, \mathrm{t}_{1}\right)=266.124, \quad \mathrm{t}_{\mathrm{w}}=0.100, \quad \mathrm{t}_{1}=0.265$

(2) When $\widetilde{c_{1}}, \widetilde{c_{2}}, \widetilde{c_{3}}$ are all triangular fuzzy numbers then

$\operatorname{TAC}\left(\mathrm{t}_{\mathrm{w}}, \mathrm{t}_{1}\right)=266.112, \quad \mathrm{t}_{\mathrm{w}}=0.100, \quad \mathrm{t}_{1}=0.266$

(3) When $\widetilde{c_{1}}, \widetilde{c_{2}}$ are triangular fuzzy numbers then,

$$
\operatorname{TAC}\left(\mathrm{t}_{\mathrm{w}}, \mathrm{t}_{1}\right)=266.162 \quad \mathrm{t}_{\mathrm{w}}=0.100, \quad \mathrm{t}_{1}=0.266
$$

(4) When $\widetilde{c_{1}}$ are triangular fuzzy numbers then,

$$
\left.\operatorname{TAC}\left(\mathrm{t}_{\mathrm{w}}, \mathrm{t}_{1}\right)\right)=266.162, \quad \mathrm{t}_{\mathrm{w}}=0.100, \quad \mathrm{t}_{1}=0.266
$$

\subsection{Sensitivity Analysis}

We now examine to sensitivity analysis of the optimal solution of the model for change in I, keeping the other parameters unchanged. The initial data from the above numerical example. 


\begin{tabular}{|l|c|c|c|c|}
\multicolumn{1}{l}{ Parameter } & \multicolumn{1}{c}{ \% of change } & \multicolumn{1}{c}{$T A C\left(\mathrm{t}_{\mathrm{w}}, \mathrm{t}_{1}\right)$} & $\mathrm{t}_{\mathrm{w}}$ & $\mathrm{t}_{1}$ \\
\hline $\mathrm{C}_{1}=250$ & -50 & 141.162 & 0.100 & 0.266 \\
\hline $\mathrm{C}_{1}=375$ & -25 & 203.662 & 0.100 & 0.266 \\
\hline $\mathrm{C}_{1}=500$ & 0 & 266.162 & 0.100 & 0.266 \\
\hline $\mathrm{C}_{1}=625$ & 25 & 328.662 & 0.100 & 0.266 \\
\hline $\mathrm{C}_{1}=750$ & 50 & 391.162 & 0.100 & 0.266 \\
\hline $\mathrm{C}_{2}=0.50$ & -50 & 258.081 & 0.100 & 0.266 \\
\hline $\mathrm{C}_{2}=0.75$ & -25 & 262.121 & 0.100 & 0.266 \\
\hline $\mathrm{C}_{2}=1.00$ & 0 & 266.162 & 0.100 & 0.266 \\
\hline $\mathrm{C}_{2}=1.25$ & 25 & 270.202 & 0.100 & 0.266 \\
\hline $\mathrm{C}_{2}=1.50$ & 50 & 274.243 & 0.100 & 0.266 \\
\hline $\mathrm{C}_{3}=1.00$ & -50 & 273.535 & 0.100 & 0.227 \\
\hline $\mathrm{C}_{3}=1.50$ & -25 & 269.855 & 0.100 & 0.251 \\
\hline $\mathrm{C}_{3}=2.00$ & 0 & 266.162 & 0.100 & 0.266 \\
\hline $\mathrm{C}_{3}=2.50$ & 25 & 262.462 & 0.100 & 0.275 \\
\hline $\mathrm{C}_{3}=3.00$ & 50 & 258.758 & 0.100 & 0.282 \\
\hline $\mathrm{C}_{4}=0.50$ & -50 & 264.458 & 0.100 & 0.269 \\
\hline $\mathrm{C}_{4}=0.75$ & -25 & 265.323 & 0.100 & 0.268 \\
\hline $\mathrm{C}_{\mathrm{w}}=1.00$ & 0 & 266.162 & 0.100 & 0.266 \\
\hline $\mathrm{C}_{4}=1.25$ & 25 & 267.000 & 0.100 & 0.264 \\
\hline $\mathrm{C}_{4}=1.50$ & 50 & 267.839 & 0.100 & 0.262 \\
\hline
\end{tabular}

\subsection{Effect, for increment parameters}

(1) $\operatorname{TAC}\left(t_{\mathrm{w}}, \mathrm{t}_{1}\right)$ increase rapidly, for increase of $\mathrm{c}_{1}$.

(2) $\mathrm{TAC}\left(\mathrm{t}_{\mathrm{w}}, \mathrm{t}_{1}\right)$ increase slowly, for increase of $\mathrm{c}_{2}$.

(3) $\mathrm{TAC}\left(\mathrm{t}_{\mathrm{w}}, \mathrm{t}_{1}\right)$ decrease slowly, for increase of $\mathrm{c}_{3}$.

(4) $\mathrm{TAC}\left(\mathrm{t}_{\mathrm{w}}, \mathrm{t}_{1}\right)$ increase slowly, for increase of $\mathrm{c}_{4}$.

\section{Conclusion}

In this paper, we have proposed a real life inventory problem in a fuzzy environment and presented solution along with sensitivity analysis approach. The inventory model developed with time depended demand, with shortages. Shortages have been allow fully backlogged in this model. Here demand rate considered as $(a+b t)$ in $0 \leq t \leq t_{w}$, and it (c-dt) in $t_{w} \leq t \leq t_{1}$. This model has been developed for single item.

In this paper, we have considered triangular fuzzy number and solved by graded mean integration method. In future, the other type of membership functions such as piecewise linear hyperbolic, L-R fuzzy number, trapezoidal fuzzy number, pentagonal fuzzy number etc can be considered to construct the membership function and then model can be easily solved. 


\section{Reference}

1. BELlMAN, R.E, AND ZADEH (1970). Decision making in a fuzzy environment, Management Science 17, B141-B164.

2. CARLSSON, C. AND P. KORHONEN (1986). A parametric approach to fuzzy linear programming, Fuzzy sets and systems, 17-30.

3. CLARK, A.J, (1992). An informal survey of multy-echelon inventory theory, naval research logistics Quarterly 19, 621-650.

4. D.Dutta and Pavan Kumar (2012). Fuzzy inventory without shortages using trapezoidal fuzzy number with sensitivity analysis IOSR Journal of mathematics, Vol. 4(3), 32-37.

5. DUTTA, D.J.R. RAO, AND R.N TIWARY (1993). Effect of tolerance in fuzzy linear fractional programming, fuzzy sets and systems 55, 133-142.

6. HAMACHER, H.LEBERLING AND H.J.ZIMMERMANN (1978). Sensitivity Analysis in fuzzy linear Programming Fuzzy sets and systems 1, 269-281.

7. HADLEY, G. AND T.M. WHITE (1963). Analysis of inventory system, PrenticeHall, Englewood Cliffs, NJ.

8. KHUN, H.W AND A.W. TUCKER (1951). Non-linear programming, proceeding second Berkeley symposium Mathematical Statistic and probability (ed) Nyman, J. University of California press 481-492.

9. Li, H.X. AND YEN, V.C. (1995). Fuzzy Sets and Fuzzy decision making, CRC press, London.

10. M.K. Maity (2008). Fuzzy inventory model with two ware house under possibility measure in fuzzy goal, Euro.J.Oper. Res 188, 746-774.

11. RAYMOND, F.E (1931). Quantity and Economic in manufacturing, McGrawHill, New York.

12. Y.Liang, F.Zhou (2011). A two warehouse inventory model for deteriorating items under conditionally permissible delay in Payment, Appl. Math. Model.35, 2221-2231.

13. ZADEH, L.A (1965). Fuzzy sets, Information and Control, 8, 338-353.

14. ZIMMERMANN, H.J. (1985). Application of fuzzy set theory to mathematical programming, Information Science, 36, 29-58.

15. ZIMMERMANN, H.J. (1987). Fuzzy sets, Decision making and expert systems, Kluwer-nijhoff publishing, Dordrecht.

16. ZIMMERMANN, H.J. (1992). "Methods and applications of Fuzzy Mathematical programming", in An introduction to Fuzzy Logic Application in Intelligent Systems (R.R. Yager and L.A. Zadeh, eds), pp.97- 120, Kluwer publishers, Boston. 